\title{
Structure and mass function of five intermediate/old open clusters
}

\author{
A. K. Durgapal and A. K. Pandey \\ State Observatory, Manora Peak, Naini Tal - 263 129, Uttaranchal, India \\ e-mail: alok, pandey@upso.ernet.in
}

Received 27 February 2001 / Accepted 30 May 2001

\begin{abstract}
The $U B V R I$ data of five northern open star clusters are used to study the mass function (MF), structure and dynamical state of these clusters. The clusters under discussion have ages ranging from $\sim 0.6$ to 5 Gyr. A comparison of the density profiles indicates that the evolution of the core of these clusters is almost the same, whereas the corona of the clusters are probably affected by the external environment and dynamical evolution. For the entire cluster region, the slope of the MF of three clusters (Be 64, Be 69 and King 5) has a value that agrees within the error with the Salpeter value, whereas King 7 and Be 20 show a steeper $(\Gamma=-2.02 \pm 0.24)$ and almost a flat MF respectively. We find that the slope of the MF of two clusters (King 5 and King 7) changes significantly from the inner region to the outer region, becoming steeper at larger radii. The dynamical relaxation time of the clusters under discussion is less than the age of the clusters, which indicates that all of these clusters are dynamically relaxed. Thus the observed mass segregation in three clusters can be attributed to the dynamical evolution of the clusters. The ratio of the clusters' present radius to the limiting radius (determined from the relation given by King 1962) can be representated by an exponent law.
\end{abstract}

Key words. galaxy: open clusters and associations: general

\section{Introduction}

Observational evidence indicates that the formation of star clusters has been efficient all along the life of our Galaxy. As a consequence, one finds star clusters with ages ranging from a few Myr to a few Gyr. This makes star clusters natural laboratories for studying star formation history in different epochs of the Galaxy, the initial mass function (IMF) of the clusters etc. The initial mass function, defined as the frequency distribution of stellar masses at the time of birth of the star clusters, is an important parameter to constrain star formation theories in star clusters. The Mass Function (MF) also plays an important role in understanding the early evolution of star clusters, because it is a fossil record of the very complex process of star formation and provides an important link between the easily observable population of luminous stars in a stellar system and the fainter, but dynamically more important, low mass stars.

Various studies indicate that the star formation process is a gradual process for a period of about $15-20 \mathrm{Myr}$ (see e.g., Pandey et al. 2000 and references therein). Therefore it is suggested that even in cases of young

Send offprint requests to: A. K. Durgapal,

e-mail: alok@upso.ernet.in open clusters we can determine only the present day mass function (PDMF). Similarly, in cases of old clusters, the MF changes with time due to stellar as well as dynamical evolutionary effects. It is also well known that in star clusters most massive stars tend to be located near the cluster center. In cases of young clusters it must be the imprint of the star formation process, whereas in old clusters it may be due to dynamical evolution of the clusters. A fundamental question in the theories of stellar and galactic evolution is whether the shape of the IMF is universal in time and space, or whether it depends on parameters like metallicity, age, environments etc. In spite of many years of continued observations, the question is still open (cf. Scalo 1986, 1998).

A knowledge of the spatial stellar mass distribution in open clusters is quite useful to study their dynamics. Detailed knowledge of the MF in different environments/different epochs of the Galaxy is crucial for studies which deal with the spectral, photometric and chemical evolution of integrated stellar systems, e.g., Pandey et al. (1989) have found that to generate integrated magnitudes and colours for old clusters, we need a steeper MF.

The aim of this paper is to analyze the structure and mass function of five intermediate/old age open clusters and to study whether the MF has any dependence on the radial distance from the cluster center. 
Table 1. Coordinates and other relevant information for the clusters under study. Distance, $E(B-V)$ and age for the clusters are taken from our earlier papers. For determining the Galactocentric distances to the clusters, a value of $8.5 \mathrm{kpc}$ has been assumed for the Galactocentric distance of the Sun.

\begin{tabular}{lcrccccc}
\hline Cluster & $\begin{array}{c}l \\
(\mathrm{deg})\end{array}$ & $\begin{array}{c}b \\
(\mathrm{deg})\end{array}$ & $\begin{array}{c}\text { Galactocentric } \\
\text { distance }(\mathrm{kpc})\end{array}$ & $\begin{array}{c}z \\
(\mathrm{pc})\end{array}$ & $\begin{array}{c}E(B-V) \\
(\mathrm{mag})\end{array}$ & $\begin{array}{c}\text { Age } \\
(\mathrm{Gyr})\end{array}$ & $\begin{array}{c}\text { Distance } \\
(\mathrm{kpc})\end{array}$ \\
\hline Be $64^{1}$ & 131.9 & 4.6 & $11.46 \pm 0.50$ & $310 \pm 40$ & $1.05 \pm 0.05$ & $0.8-1.0$ & $3.88 \pm 0.48$ \\
Be $69^{1}$ & 174.4 & -1.8 & $11.35 \pm 0.30$ & $-90 \pm 10$ & $0.65 \pm 0.05$ & $0.8-1.0$ & $2.86 \pm 0.31$ \\
King $7^{2}$ & 149.7 & 1.0 & $10.46 \pm 0.34$ & $40 \pm 8$ & $1.25 \pm 0.05$ & $0.6-0.8$ & $2.20 \pm 0.34$ \\
King 5 & 143.7 & -4.3 & $10.09 \pm 0.25$ & $-140 \pm 10$ & $0.82 \pm 0.05$ & 1.0 & $1.90 \pm 0.10$ \\
Be $20^{3}$ & 203.5 & -17.3 & $17.11 \pm 0.48$ & $-2700 \pm 120$ & $0.10 \pm 0.05$ & 5 & $9.03 \pm 0.48$ \\
\hline
\end{tabular}

1 Pandey et al. (1997).

2 Durgapal et al. (1997).

3 Durgapal et al. (2001).

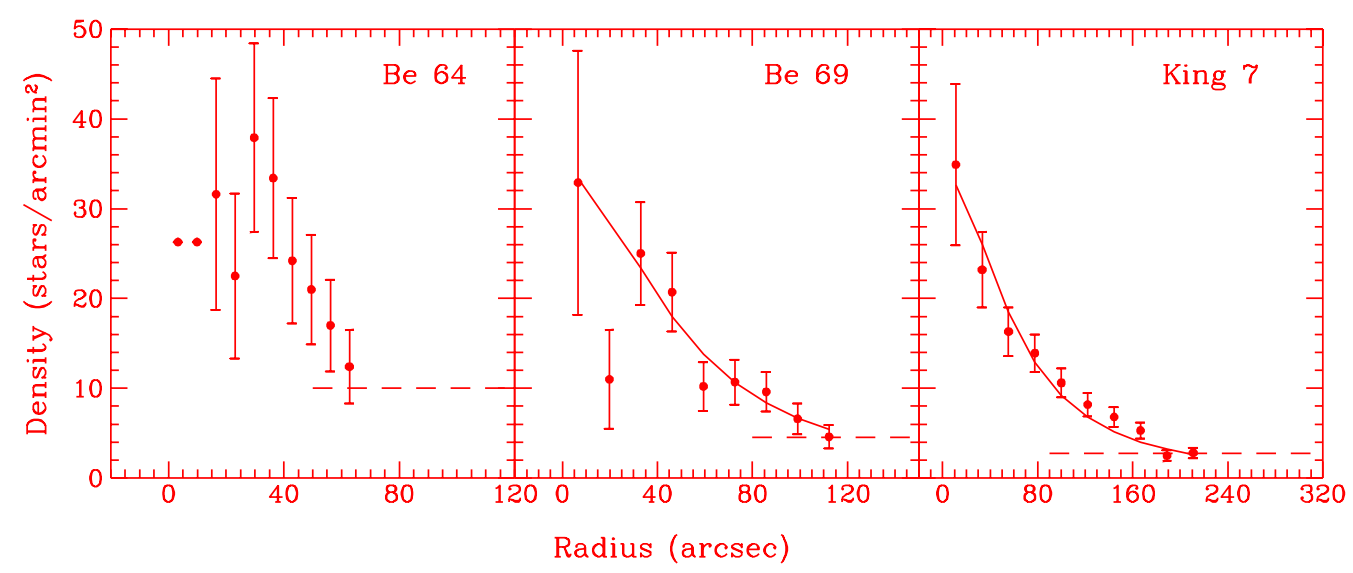

Fig. 1. Radial density profile for the clusters Be 64, Be 69 and King 7. Solid curve shows a least squares fit of the King (1962) profile to the observed data points. The error bars represent $\frac{1}{\sqrt{N}}$ errors. The data point at $r=19.8^{\prime \prime}$, of cluster Be 69 , was excluded while fitting the King profile. The dashed line shows the density of the field stars in the cluster region.

\section{Data}

The UBVRI photometric observations for the clusters Be 64, Be 69, King 5, King 7 and BVRI observations for the cluster Be 20 were carried out using the photometric CCD system at the f/13 Cassegrain focus of the 104-cm Sampurnanand reflector of the State Observatory, Naini Tal, from November 1990-November 1996. The CCDs having size $384 \times 576$ pixel $^{2}$ (for Be 64 and Be 69) and $1024 \times 1024$ pixel $^{2}$ (for King 7, King 5 and Be 20) were used for the observations of the clusters. In order to improve the $\mathrm{S} / \mathrm{N}$ ratio, the observations were taken in binning mode of $2 \times 2$ pixels. In this setup each pixel of $384 \times 576$ and $1024 \times 1024$ pixel $^{2}$ CCDs corresponds to 0.66 arcsec and 0.7 arcsec respectively, whereas these chips cover a field of $2 \times 3$ and $6 \times 6$ arcmin $^{2}$ respectively. Nearby field regions at $\sim 30^{\prime}$ away from the cluster center were also observed to estimate the contamination due to the field stars in the cluster region. These fields are located to the East and West for Be 64 and North, South for Be 69 and King 7. For the cluster King 5 the nearby field region was observed towards the East. The region away from the cluster extent was considered as a field region in the case of Be 20. The details can be found in Pandey et al. (1997), Durgapal et al. (1997) and Durgapal et al. (2001).

The stellar photometric routine of DAOPHOT was used for the magnitude determination. These magnitudes were calibrated using Landolt $(1983,1992)$ photoelectric standards. The details of the observations and results obtained are described in our previous papers (Pandey et al. 1997; Durgapal et al. 1997; Durgapal et al. 2001). Various parameters obtained for the clusters under discussion are summarized in Table 1. The photometric data used in the ensuing sections have also been given in the above mentioned papers.

\section{Projected radial surface density}

Since the observed frames covers a sufficiently large area, the present data can be used to estimate the extent as well as the radial surface density distribution of the cluster. The cluster center was derived iteratively by 


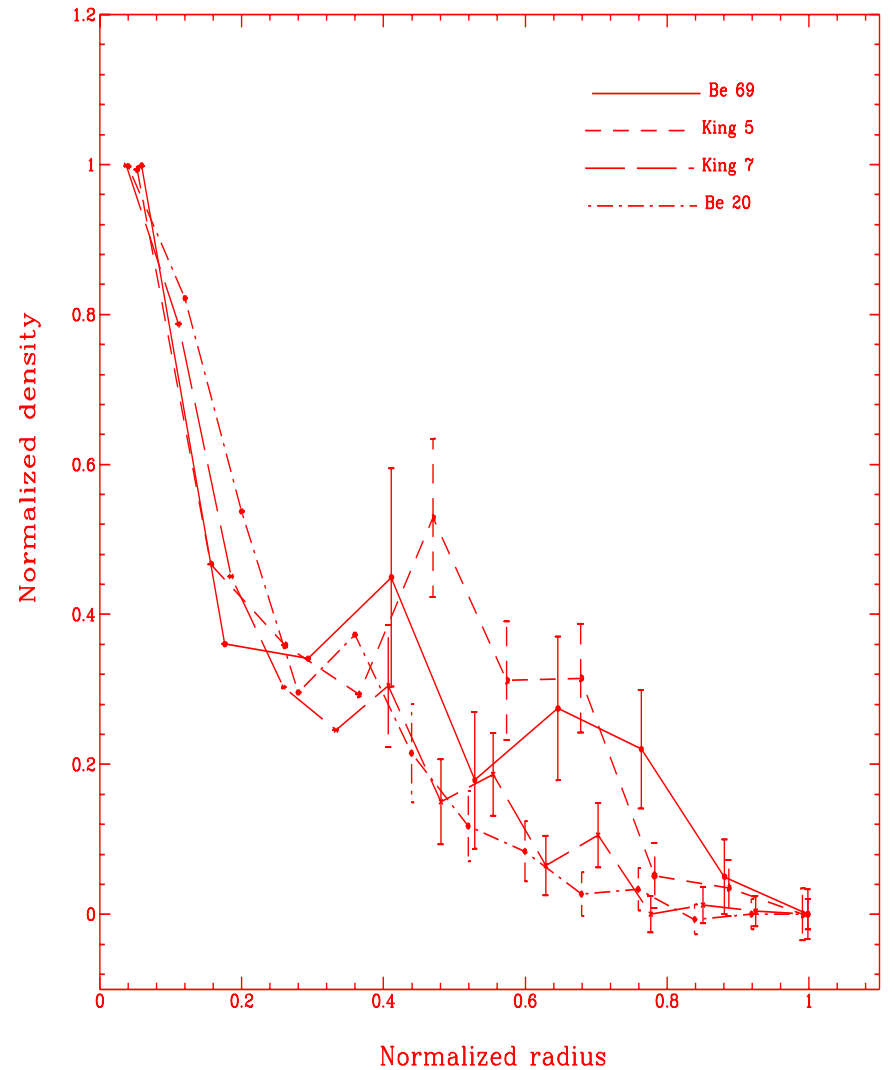

Fig. 2. Normalized radial density profiles of the clusters Be 69, King 7, King 5 and Be 20.

calculating average $X$ and $Y$ positions of stars from an eye-estimated center, until it converged to a constant value. The $X$ and $Y$ positions of the stars for the clusters Be 64 \& Be 69, King 7 and King 5 \& Be 20 have been taken from Pandey et al. (1997), Durgapal et al. (1997) and Durgapal et al. (2001) respectively. The $(X$, $Y)$ pixel coordinates of the cluster center are $(100,130)$, $(230,150),(213,213),(227,205)$ and $(224,220)$ for Be 64 , Be 69, King 7, King 5 and Be 20 respectively. An error of a few pixels is expected in locating the cluster center. The radial surface density in various concentric annuli was obtained by dividing the number of stars in each annulus by the area of that annulus. Annulus sectors were considered in outermost regions where it was not possible to deduce the radial surface density from full annuli. The error was calculated by Poisson Statistics. The radial surface densities as a function of radial distance are shown in Fig. 1. The radial density profile of individual clusters is discussed below.

\section{Be 64}

An identification map of the cluster Be 64 shows a faint cluster that emerges weakly from the field background. The stellar density profile for Be 64 indicates an irregular structure and does not show a density peak at the center, as do most of the clusters. The density of stars is less in the central region. This type of feature has also been observed in a few intermediate/old age clusters (e.g., Haffner 6, Patat \& Carraro 1995; Tombaugh 1, Carraro \& Patat 1995; NGC 7044, Aparicio et al. 1993). Lack of stars in the central region of the cluster poses an interesting question regarding the evolution of star clusters. Since the area imaged is not large enough, we estimated the extent of the cluster by extrapolating the radial density profiles. The extrapolation of the density profile reaches the field density level at $\sim 75^{\prime \prime}$ which corresponds to a linear size of $\sim 1.4$ pc.

\section{Be 69}

The radial density profile of the cluster Be 69 shows a rather systematic variation, barring a dip in the density at $\sim 20^{\prime \prime}$. The stellar density decreases with increasing radial distance from the cluster center and drops to the field star density, obtained from observations of nearby field regions. The density profile can be representated by a King (1962) profile having $f_{0}=34.3 \pm 1.9$ and $r_{\mathrm{c}}=499^{\prime \prime} 3 \pm 33^{\prime \prime} .9$. The density profile for the cluster indicates a radial extent of $\sim 110^{\prime \prime}$ for the cluster Be 69 which corresponds to a linear size of $\sim 1.5 \mathrm{pc}$.

\section{King 7}

The stellar densities in the cluster King 7 show a systematic variation with radial distance from the cluster center and can be represented by a King (1962) profile which gives the best fit parameter as $f_{0}=32.7 \pm 1.8$ and $r_{\mathrm{c}}=63 .{ }^{\prime \prime} 9 \pm 5 . .5$. At radius $\sim 175^{\prime \prime}$ the density of the cluster region becomes approximately equal to the field star density, therefore, the radial extent of the cluster is estimated as $\sim 175^{\prime \prime}$, which corresponds to a linear size of about $1.9 \mathrm{pc}$ at the distance of the cluster.

\section{King 5 and $\mathrm{Be} 20$}

The stellar density profiles of the clusters King 5 and Be 20 have been discussed in a previous paper (cf. Durgapal et al. 2001). The density profile of King 5 indicates that the cluster is extended up to $170^{\prime \prime}$ which corresponds to a linear radius of about $1.6 \mathrm{pc}$, whereas the cluster Be 20 is extended up to $\sim 150^{\prime \prime}$, corresponding to a linear radius of about $6.6 \mathrm{pc}$.

To calculate the surface density in the outskirts of the cluster we have to use annulus sectors. The radial distances after which annulus sector were used are $55^{\prime \prime}, 150^{\prime \prime}, 160^{\prime \prime}$ and $157^{\prime \prime}$, respectively for the clusters Be 64, King 7, King 5, and Be 20.

To compare density profiles of the clusters under discussions, we normalized the densities and the radial extent of the clusters. The normalized main-sequence stellar density profiles of the clusters, barring Be 64 , are shown in Fig. 2. The cluster Be 64 is excluded as it shows an irregular radial density profile. Figure 2 suggests that the evolution of the core of the clusters under discussion is 


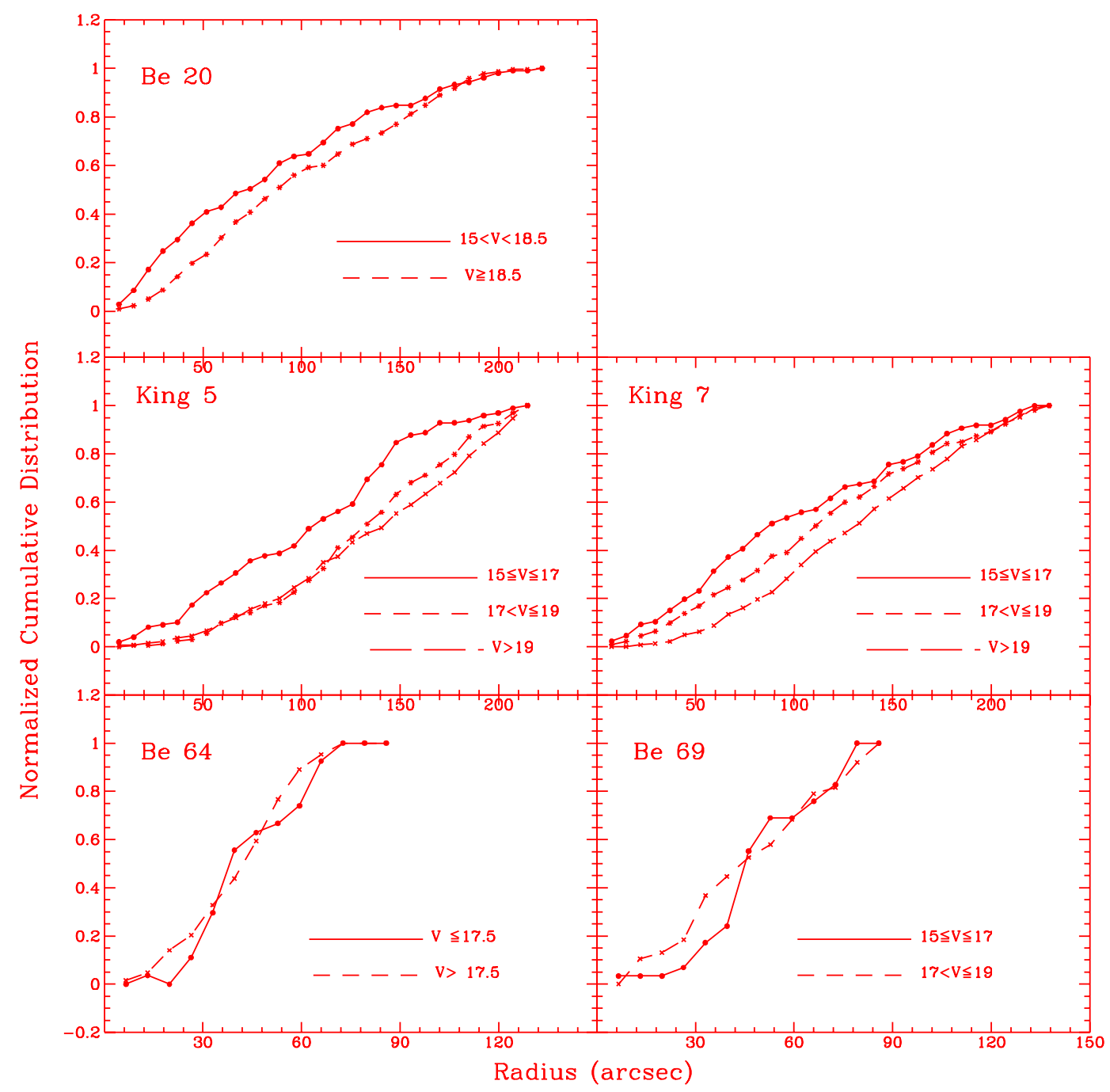

Fig. 3. Cumulative radial distribution of stars in different luminosity ranges.

similar whereas the structure of the corona of the cluster differs. This may be due to various reasons e.g., (i) the dynamical relaxation, which causes mass segregation in the cluster (Sect. 4), (ii) the external environment, as we know that the corona of the clusters are moulded by the galactic tidal fields (Mathieu 1985). A large sample of clusters is needed to study in detail the evolution of their coronae.

\section{Mass segregation}

There is much evidence that indicates that the mean stellar mass in star clusters is a decreasing function of radial distance (see e.g., Sagar et al. 1988; Pandey et al. 1992; Meylan G. 2000 and references therein). The structure of a cluster can be affected by the mass segregation in the clusters. In intermediate and old open clusters, the mass segregation occurs due to dynamical relaxation of the cluster. To study the mass segregation in the sample of clusters under discussion, we constructed cumulative radial stellar distribution of stars for two or three luminosity ranges depending upon the range in brightness and statistics in the available data. The cumulative radial distribution (CRD) for different brightness levels (i.e., different mass levels) is shown in Fig. 3.

The cluster Be 64 is a poor cluster and we could divide the sample for two brightness levels only i.e., $V \leq 17.5$ and $V>17.5$. The bright sample contains mainly giant stars. A Kolmogorov-Smirnov (KS) test indicates that these two CRDs belong to same population i.e., there is no evidence for mass segregation. For the cluster Be 69, the data is divided for $15 \leq V \leq 17$ and $17<V \leq 19$. These samples mainly contain MS stars. The KS test gives weak evidence (confidence level 0.7) for mass segregation although in a reverse sense i.e., low mass stars are more centrally concentrated as compared to massive stars. If it is real it indicates either that equipartition of the energy has not completely taken place as expected due to dynamical relaxation of the clusters, or that the stellar distribution is severely affected by the galactic tidal field.

The data for King 5 and King 7, divided for luminosity ranges $15 \leq V \leq 17,17<V \leq 19$ and $V>19$, give 
Table 2. Details of artificial add star experiment. The $N_{\mathrm{m}}$, $N_{\mathrm{i}}$ and $N_{\mathrm{f}}$ are the number of stars measured in original CCD frames, the number of stars inserted and the number of artificial star frames generated from a given original CCD frame, respectively. The number of stars added to each frame is simply $N_{\mathrm{i}} \sum N_{\mathrm{f}} . \Delta m$ is the magnitude range of artificial stars added to the frame.

\begin{tabular}{lccccc}
\hline \multirow{2}{*}{ Region } & \multirow{2}{*}{ Filter } & $N_{\mathrm{m}}$ & $N_{\mathrm{i}}$ & $\Delta m$ & $N_{\mathrm{f}}$ \\
& & & & & \\
\hline King 5 & $V$ & 870 & 130 & $14.0-20.0$ & 2 \\
& & & & $15.0-20.0$ & 2 \\
& & & & $16.0-20.0$ & 2 \\
& & & & $17.0-20.0$ & 2 \\
& & & & $18.0-20.0$ & 2 \\
& & & & $19.0-20.0$ & 2 \\
\hline King 7 & $V$ & 736 & 110 & $14.0-20.0$ & 2 \\
& & & & $15.0-20.0$ & 2 \\
& & & & $16.0-20.0$ & 2 \\
& & & & $17.0-20.0$ & 2 \\
& & & & $18.0-20.0$ & 2 \\
& & & & $19.0-20.0$ & 2 \\
\hline Be 20 & & & & $18.0-21.0$ & 2 \\
& & & & $18.5-21.0$ & 2 \\
& & & & $19.0-21.0$ & 2 \\
& & & & $20.5-21.0$ & 2 \\
& & & $20.5-21.0$ & 2 \\
& & & & 2 \\
\hline
\end{tabular}

better evidence in the case of King 7 (confidence level $\sim 0.9$ for the samples $15 \leq V \leq 17$ and $17<V \leq 19$; and $\sim 0.99$ for the samples $15 \leq V \leq 17$ and $V>19)$ and strong evidence for the cluster King 5 (confidence level $\sim 0.99$ ) for mass segregation, in the sense that massive stars are more centrally concentrated.

The cluster Be 20 is the oldest object ( $\sim 5$ Gyr) amongst the clusters under discussion. The blue turnoff is estimated at $V \sim 18.2$. The data is divided for two luminosity ranges, $15 \leq V<18.5$ and $V \geq 18.5$. The brighter luminosity sample contains evolved stars whereas the fainter sample contains only MS stars. The CRD for these two samples is shown in Fig. 3 where mass segregation is clearly evident (confidence level $\sim 0.98$ ).

A comparison of the CRDs of the clusters King 5, King 7 and Be 20, where the mass segregation is evident, indicates an interesting feature. The CRD curves of brighter stars, in case of King 5 and Be 20, are significantly different than those of fainter stars over the entire cluster region; however the CRDs of the King 5 for the fainter levels (i.e., $17<V \leq 19$ and $V>19$ ) are indistinguishable. For King 7 the CRDs of the brighter stars $(15 \leq V \leq 17)$ and stars having $17<V \leq 19$ are different (confidence level 0.9) for $r \leq 140^{\prime \prime}$ and for the rest of the cluster region i.e., $140^{\prime \prime} \leq r \leq 175^{\prime \prime}$ the two distributions are similar. Whereas the CRD of the stars having
$V>19$ can be discriminated from the CRDs of brighter stars and stars having $17<V \leq 19$ for the entire cluster region at a confidence level 0.99 and 0.98 respectively. On the basis of the above discussions it seems that the mass segregation has taken place over the entire cluster region in case of King 5 and Be 20, whereas in King 7 the equipartition of energy is still in progress. The above conclusion is also supported by the fact that King 7 has a lower age $(\sim 0.6 \mathrm{Gyr})$ compared to King $5(\sim 1 \mathrm{Gyr})$ and Be 20 ( 5 Gyr).

\section{Mass function}

\subsection{Completeness of the data}

A quantitative evaluation of completeness of the photometric data with respect to the brightness and the position on a given frame is necessary because it is required to convert an observed luminosity function (LF) to a true LF. The method to determine the completeness factor, $\mathrm{CF}$, of the stellar photometric data in a CCD frame using the DAOPHOT software package has been discussed by several authors (see e.g., Stetson 1987; Mateo 1988; Sagar \& Griffiths 1998b and references therein). Two passbands, such as $B$ and $V$ or $V$ and $I$, are required for identification of a significantly different mixture of stellar populations (i.e., field stars) which are superimposed on the cluster fields. It is therefore required to construct the LF either from a $V,(B-V)$ diagram or from $V,(V-I)$ diagram instead of from a single $B, V$ or $I$ passband. We preferred the $V,(V-I)$ digram over the $V,(B-V)$ diagram as it is generally deeper at least by a magnitude. The main disadvantage of using two passbands for the construction of the LF is that both passbands introduce further incompleteness, and determination of incompleteness in both passbands is a cumbersome process. Different approaches have been given by various authors to deal with such a situation. For example, Mateo (1988) assumed that the star counts in the two bands are independent and adopted a completeness factor as the product of the CF values of the two frames. Sagar \& Richtler (1991) adopted the minimum value of the completeness factors of the pair to correct the star counts. They argue that the two frames are not independent and that the multiplicative assumption of Mateo (1988) could not be justified. A detailed discussion on the topic is given in Sagar \& Griffiths (1998b). We adopted the approach given by Sagar \& Griffiths (1998b). Briefly, the method consists of insertion of randomly selected artificial stars with known magnitude and positions in the original $V$ image frame. In the case of the $I$ band image, the inserted stars have the same geometrical positions but differ in $I$ brightness according to mean $(V-I)$ colour of the main sequence stars. The frames are re-reduced using the same procedure used for the original frames. The ratio of recovered to inserted stars in the different magnitude bins in a defined spatial region directly gives the completeness factor, CF, for that region as a function of magnitude. About 10-15 percent of the number of actually detected 
Table 3. Variation of the completeness factor (CF) with the MS brightness in both cluster and field regions. The completeness factor in different regions of the clusters is also given (for details see Sect. 2 of the text).

\begin{tabular}{|c|c|c|c|c|c|}
\hline cluster & $\begin{array}{l}V \text { mag } \\
\text { Range }\end{array}$ & \multicolumn{3}{|c|}{$\begin{array}{c}\text { Cluster region } \\
(\mathrm{CF})\end{array}$} & $\begin{array}{l}\text { Field region } \\
\qquad(\mathrm{CF})\end{array}$ \\
\hline \multirow{7}{*}{ King 5} & \multirow{7}{*}{$\begin{array}{l}14-15 \\
15-16 \\
16-17 \\
17-18 \\
18-19 \\
19-20 \\
\end{array}$} & $r<80^{\prime \prime}$ & $80^{\prime \prime}<r<170^{\prime \prime}$ & whole frame & \multirow{7}{*}{$\begin{array}{l}1.00 \\
1.00 \\
1.00 \\
0.99 \\
0.98 \\
0.98\end{array}$} \\
\hline & & 1.00 & 1.00 & 1.00 & \\
\hline & & 1.00 & 1.00 & 1.00 & \\
\hline & & 1.00 & 1.00 & 1.00 & \\
\hline & & 0.91 & 0.96 & 0.96 & \\
\hline & & 0.88 & 0.94 & 0.94 & \\
\hline & & 0.80 & 0.86 & 0.84 & \\
\hline \multirow{7}{*}{ King 7} & & $r<60^{\prime \prime}$ & $60^{\prime \prime}<r<175^{\prime \prime}$ & whole frame & \multirow{7}{*}{$\begin{array}{l}1.00 \\
1.00 \\
1.00 \\
0.98 \\
0.93 \\
0.90\end{array}$} \\
\hline & $14-15$ & 1.00 & 1.00 & 1.00 & \\
\hline & $15-16$ & 1.00 & 1.00 & 1.00 & \\
\hline & $16-17$ & 0.99 & 1.00 & 0.99 & \\
\hline & $17-18$ & 0.93 & 0.98 & 0.98 & \\
\hline & $18-19$ & 0.89 & 0.95 & 0.93 & \\
\hline & $19-20$ & 0.83 & 0.93 & 0.90 & \\
\hline \multirow{7}{*}{ Be 20} & & Whole cluster & Field region & & \\
\hline & $18-18.5$ & 1.00 & 1.00 & & \\
\hline & $18.5-19$ & 1.00 & 1.00 & & \\
\hline & $19-19.5$ & 1.00 & 1.00 & & \\
\hline & $19.5-20$ & 0.92 & 0.98 & & \\
\hline & $20-20.5$ & 0.90 & 0.95 & & \\
\hline & $20.5-21$ & 0.83 & 0.88 & & \\
\hline
\end{tabular}

stars are inserted at one time, so that the crowding characteristics of the original data remain almost unchanged. The luminosity distribution of the artificial stars has been chosen in such a way that more stars are inserted into the fainter magnitude bins.

Various parameters, like stars added, stars recovered and the completeness factor $\mathrm{CF}$ for three clusters, namely King 5, King 7 and Be 20, as well as for nearby field regions have been obtained and these are given in Tables 2 and 3 . In the case of cluster Be 20 the region outside the extent of the cluster is considered as field region. The clusters Be 64 and Be 69 are poor and crowding is not severe, therefore we did not apply the incompleteness corrections assuming that the effect of incompleteness is the same for both the cluster and the field regions.

\subsection{Determination of mass function}

As discussed earlier, the LF of a cluster can be obtained from the colour-magnitude diagram and it can be converted to the mass function (MF). To derive the MF from the LF, we need theoretical evolutionary tracks and accurate knowledge of the cluster parameters such as reddening, distance, age etc. Theoretical models by Bertelli et al. (1994) were used to convert the observed LF to the MF. These evolutionary tracks are derived from stellar models which include the effects of mass loss and convective core overshooting.

To derive the true LF of the cluster we have to remove contamination due to field stars. The most reliable method to decide cluster membership is proper motion and spectroscopic studies. Since these studies are not available for the present sample, we used only the photometric criterion to remove the field star contamination from the sample. We proceeded in the following way. We defined a blue and red envelope for the main-sequence on $V,(V-I)$ CMD for stars lying within the cluster boundary (see e.g., Durgapal et al. 1997). The same envelopes were drawn on the corresponding field region CMDs. Star counts as a function of luminosity were made in both (cluster and field) regions. After normalizing the area we can estimate the number of field stars present in various magnitude bins of the cluster region. The observed LF of the cluster and the field regions were also corrected for data incompleteness. The true LF for the cluster was obtained by subtracting the 
Table 4. Luminosity functions for the clusters used in the present study. $N_{\mathrm{C}}$ and $N_{\mathrm{F}}$ denote MS star counts for the cluster and nearby field region in various $V$ magnitude bins. $N$ is the number of probable cluster stars after applying field star and data incompleteness corrections (for details see text).

\begin{tabular}{|c|c|c|c|c|}
\hline cluster & $\begin{array}{c}\text { Range in } \\
V \text { mag }\end{array}$ & $N_{\mathrm{C}}$ & $N_{\mathrm{F}}$ & $N$ \\
\hline \multirow[t]{6}{*}{ Be 64} & $17.0-17.5$ & 3 & - & 3 \\
\hline & $17.5-18.0$ & 8 & 1 & 7 \\
\hline & $18.0-18.5$ & 10 & 2 & 8 \\
\hline & $18.5-19.0$ & 8 & - & 8 \\
\hline & $19.0-19.5$ & 11 & 2 & 9 \\
\hline & $19.5-20.0$ & 11 & 3 & 8 \\
\hline \multirow[t]{5}{*}{ Be 69} & $15.5-16.0$ & 5 & 1 & 4 \\
\hline & $16.0-17.0$ & 13 & 2 & 11 \\
\hline & $17.0-18.0$ & 20 & 6 & 14 \\
\hline & $18.0-19.0$ & 23 & 7 & 16 \\
\hline & $19.0-20.0$ & 19 & 6 & 13 \\
\hline \multirow[t]{5}{*}{ King 5} & $15.5-16.0$ & 16 & 2 & 14.0 \\
\hline & $16.0-17.0$ & 27 & 2 & 25.0 \\
\hline & $17.0-18.0$ & 36 & 12 & 25.4 \\
\hline & $18.0-19.0$ & 68 & 13 & 59.0 \\
\hline & $19.0-20.0$ & 54 & 35 & 28.6 \\
\hline \multirow[t]{5}{*}{ King 7} & $15.5-16.0$ & 10 & 1 & 9.0 \\
\hline & $16.0-17.0$ & 29 & 3 & 26.3 \\
\hline & $17.0-18.0$ & 53 & 9 & 44.7 \\
\hline & $18.0-19.0$ & 99 & 23 & 81.7 \\
\hline & $19.0-20.0$ & 99 & 26 & 81.1 \\
\hline \multirow[t]{6}{*}{ Be 20} & $18.0-18.5$ & 24 & - & 24.0 \\
\hline & $18.5-19.0$ & 32 & 6 & 26.0 \\
\hline & $19.0-19.5$ & 27 & 6 & 21.0 \\
\hline & $19.5-20.0$ & 33 & 6 & 29.8 \\
\hline & $20.0-20.5$ & 33 & 8 & 28.3 \\
\hline & $20.5-21.0$ & 15 & 2 & 15.8 \\
\hline
\end{tabular}

Table 5. The slope of the mass function derived from the luminosity functions given in Table 4 .

\begin{tabular}{ccccc}
\hline Cluster Mass range & \multicolumn{2}{c}{ Mass } \\
& $\left(M_{\odot}\right)$ & $\begin{array}{c}\text { Function } \\
\text { Inner } \\
\text { region }\end{array}$ & $\begin{array}{c}\text { Outer } \\
\text { region }\end{array}$ & $\begin{array}{c}\text { Whole } \\
\text { cluster }\end{array}$ \\
\hline \multirow{2}{*}{ Be 64} & $1.20-1.9$ & - & - & $-1.05 \pm 0.20$ \\
& $1.35-1.9$ & - & - & $-1.36 \pm 0.15$ \\
\cline { 2 - 5 } & $0.90-1.9$ & - & - & $-1.06 \pm 0.36$ \\
Be 69 & & & - & $-1.67 \pm 0.22$
\end{tabular}

King $7 \quad 1.10-2.4 \quad-0.48 \pm 0.82-2.62 \pm 0.14-2.02 \pm 0.24$

King 5

$0.90-1.8+1.82 \pm 0.98-1.7 \pm 0.8-1.07 \pm 0.66$

\begin{tabular}{lllll}
$1.06-1.8$ & - & - & $-1.69 \pm 0.91$ \\
\hline
\end{tabular}

Be 20

$0.80-1.2+0.19 \pm 1.0+1.28 \pm 0.9+0.69 \pm 1.01$

$0.90-1.2$

$-0.82 \pm 0.82$

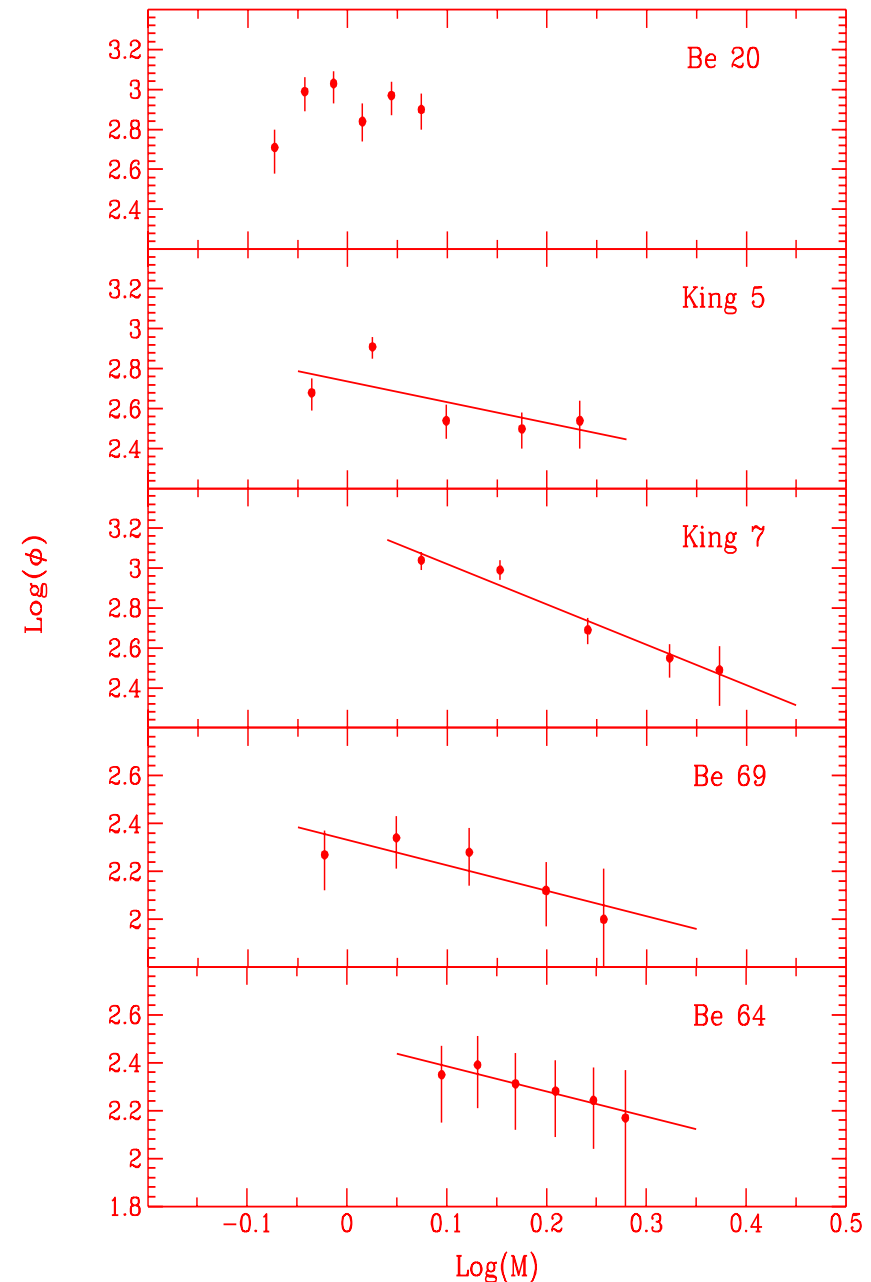

Fig. 4. Mass functions for the clusters under discussion. The error bars represent $\frac{1}{\sqrt{N}}$ errors.

observed LF of the field region from the observed LF of the cluster region. The true LFs obtained for the clusters under discussions are given in Table 4.

The mass function is expressed as a power law, $N(\log m) \propto m^{\Gamma}$ and the slope of the mass function is given as ;

$\Gamma=\frac{\mathrm{d} \log N(\log m)}{\mathrm{d} \log m}$

where $N(\log m)$ is number of stars per unit logarithmic mass interval. The Salpeter (1955) value for the slope of the IMF is $\Gamma=-1.35$. The LFs given in Table 4 were converted to the MFs using the theoretical models of Bertelli et al. (1994) and the resulting MFs are plotted in Fig. 4. The values of the MF slope $\Gamma$ along with the mass range and errors are given in the last column of Table 5 where the quoted errors are errors resulting from the linear least squares fit to the data points. Three clusters, namely Be 64, Be 69, and King 5, have a MF slope in agreement, within error, with the Salpeter value. 
A. K. Durgapal and A. K. Pandey: Structure and mass function of five intermediate/old open clusters

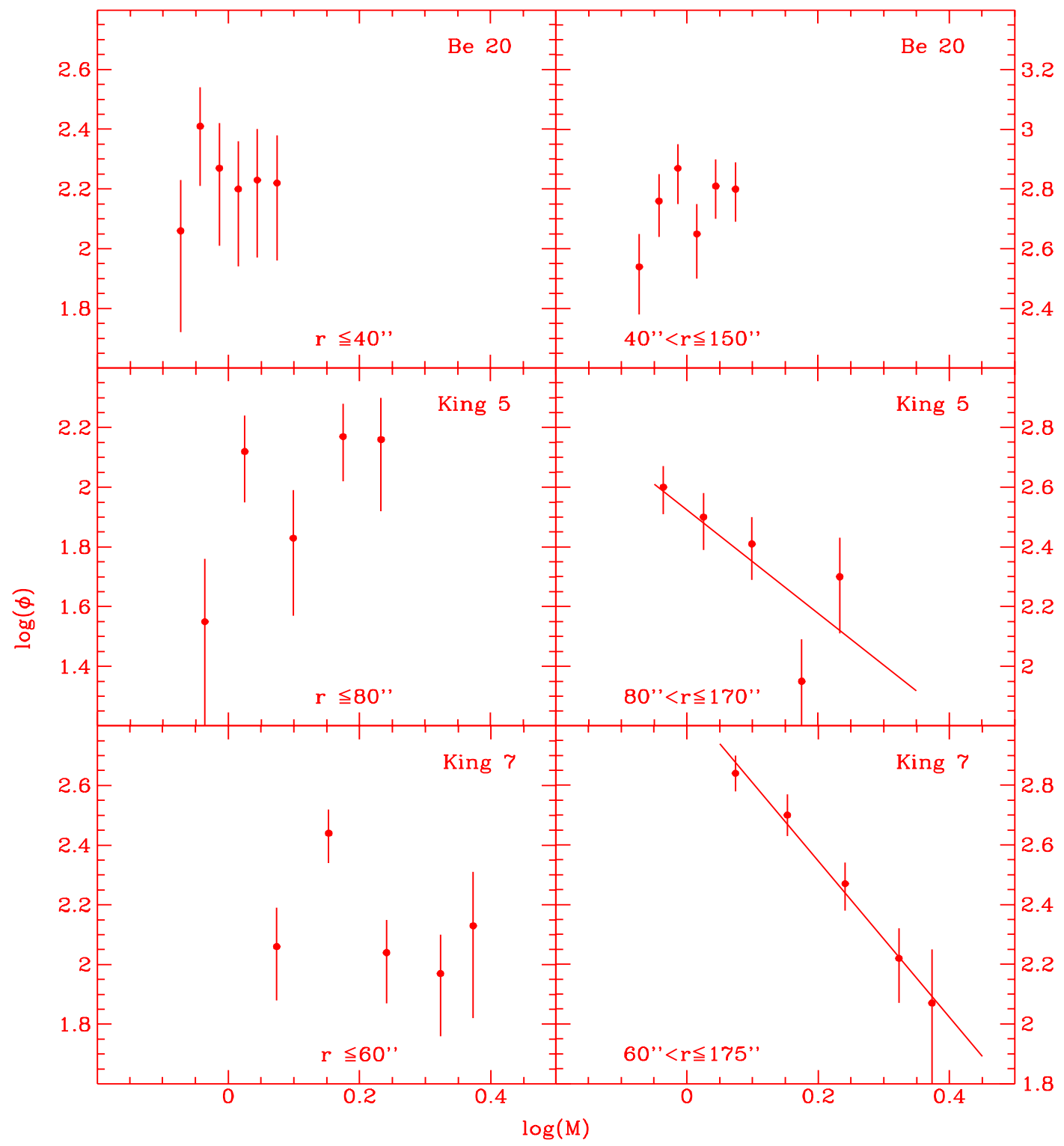

Fig. 5. Spatial variation of mass function slope for three clusters King 7 , King 5 and Be 20. The error bars represent $\frac{1}{\sqrt{N}}$ errors.

The cluster King 7 shows a steeper slope of the MF whereas the slope for Be 20 is almost flat.

In the literature there is evidence for spatial variation of MF within a cluster (see e.g., Pandey et al. 1992 and references therein). The data for three clusters (King 5, King 7 and Be 20) were divided into subregions to investigate spatial variation of the MF within the clusters. The data for Be 64 and Be 69 are not sufficient to study the spatial variation of the MF. The MFs obtained in the subregions of the clusters are shown in Fig. 5, which indicate a noisy $\mathrm{MF}$ in the central region of all three clusters. The outer region of the cluster King 7 shows a steeper slope $(\Gamma=-2.62 \pm 0.14)$ than that obtained for the outer region of King $5(\Gamma=-1.7 \pm 0.8)$ whereas the MF is rather flat in the outer region of the cluster Be 20. The MF of Be 20, in the inner as well as outer region, shows a truncation at $\sim 0.9 M_{\odot}$. The MF in the inner region of the cluster King 5 also indicates a turnover in the $\mathrm{MF}$ at $\sim 1.0 M_{\odot}$. These features seem to be realistic as we have corrected the data for incompleteness, which is not very severe $(\sim 0.8)$.

The MFs of the clusters King 5, King 7 and Be 20 seem to be affected by the mass segregation. Be 20 has a flat MF akin to MFs of older clusters, as reported in previous studies e.g., King 2 (Aparicio et al. 1990) and NGC 6791 (Kaluzny \& Rucinski 1995). It is interesting to note that the MF slopes for the inner as well as outer regions of the cluster Be 20 are flat. The MFs of the clusters King 5 and the Be 20 (entire cluster region), shown in Fig. 4, indicate a turnover at $\sim 1 M_{\odot}$. It is difficult to access whether the turnover is due to the initial mass function (IMF) or it 
is a result of loss of low mass stars from the clusters by various dynamical processes.

\section{Dynamical state of the clusters}

Dynamical relaxation is one of the possible reasons for spatial variations in the slope of the mass function. At the time of formation, the cluster may have a uniform spatial stellar mass distribution, which may be modified due to dynamical evolution of the clusters. Because of dynamical relaxation, low mass stars in a cluster may possess large random velocities; consequently, they will try to occupy a larger volume than the high mass stars (cf. Mathieu \& Latham 1986; McNamara \& Sekiguchi 1986; Mathieu 1985). Thus, mass segregation develops in the time scale required to exchange energy between stars of different mass by scattering. The dynamical relaxation time, $T_{\mathrm{E}}$, is the time in which the individual stars exchange energies and their velocity distribution approaches a Maxwellian equilibrium. $T_{\mathrm{E}}$ for the present sample is estimated using the relation

$T_{\mathrm{E}}=\frac{8.9 \times 10^{5} N^{1 / 2} R_{\mathrm{h}}^{3 / 2}}{\bar{m}^{1 / 2} \log (0.4 N)}$

where $N$ is the number of cluster members, $R_{\mathrm{h}}$ is the radius containing half of the cluster mass and $\bar{m}$ is the average mass of the cluster stars (Spitzer \& Hart 1971). The number of probable MS stars is estimated using the CMDs of the clusters after subtracting the contribution due to field stars and applying the necessary correction for data incompleteness. Giant branch stars are counted individually and added to the MS stars to get the total number, $N$, of cluster stars. The total mass of the cluster in a given mass range can be obtained from the following relation

$M_{\text {cluster }}=C \int_{M_{\mathrm{L}}}^{M_{\mathrm{U}}} M^{\Gamma} M \mathrm{~d} M$

where $M_{\mathrm{U}}$ and $M_{\mathrm{L}}$ are the upper and lower limits for stellar masses in the cluster and $\Gamma$ is the slope of the MF. The contribution of MS stars in the total mass of the cluster was estimated using Eq. (3). The mass of individual giant stars was also estimated and was added to the mass of MS stars to get total mass of the cluster. Due to our inability to estimate the $R_{\mathrm{h}}$ from the present data, we assume that the $R_{\mathrm{h}}$ is equal to half of the cluster radius listed in Table 6. Various parameters thus obtained are also given in Table 6 . The angular values are converted to linear values using the cluster distance listed in Table 1. A comparison of the cluster age with its relaxation time obtained in the present study indicates that the relaxation time is smaller than the age of the clusters. Thus we can infer that all the clusters under discussion are dynamically relaxed.

The limiting radius of a cluster moving in the general force field of the Galaxy is defined as the distance from the cluster center at which the attraction of a given star

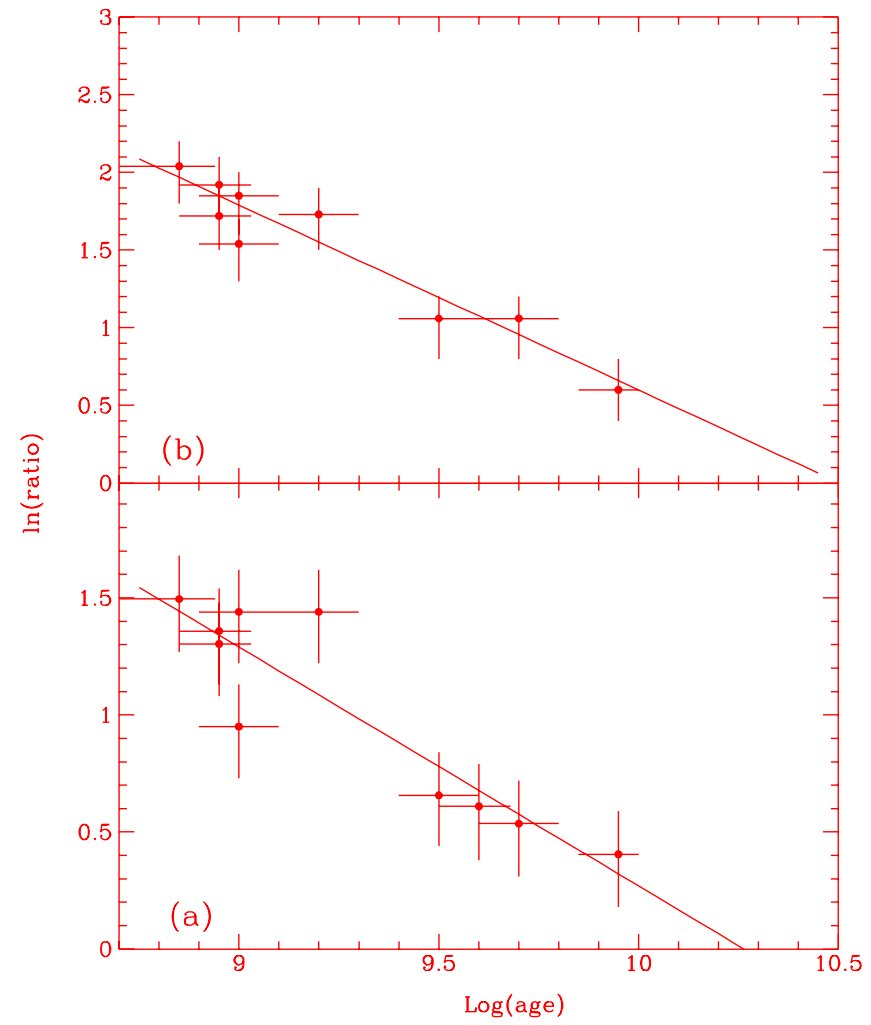

Fig. 6. a) Variation of ratio (limiting radius/estimated radius) with cluster age. Only observed stellar masses are considered in calculating the limiting radius. b) Same as above but unseen mass (up to the lowest stellar mass $0.1 M_{\odot}$ ) is also considered in calculating the limiting radius.

to the cluster is balanced by the attraction of external masses (Kholopov 1969). The limiting radius of a bound cluster can be determined from the relation given by King (1962)

$r_{\text {lim }}=R_{\mathrm{p}}\left(M_{\text {cluster }} / 3.5 M_{\mathrm{G}}\right)^{1 / 3}$

where $R_{\mathrm{p}}$ is the perigalactic distance of the cluster and $M_{\mathrm{G}}$ is the mass of the Galaxy. We calculated the limiting radius for the clusters under discussion as well as for another five clusters namely Tr 5, NGC 6791, Be 81, Be 99 and NGC 7044, for which data were taken from the literature. The $R_{\mathrm{p}}$ is taken as galactocentric distance of the cluster and the mass of the Galaxy $M_{\mathrm{G}} \sim 2 \times 10^{11}$. The limiting radius obtained for these clusters is given in Table 6 .

The ratio of the limiting radius $\left(r_{\lim }\right)$ to the present radius of the cluster $\left(r_{\text {pre }}\right)$ as a function of age is shown in Fig. 6a. The $r_{\text {pre }}$ for the clusters discussed in the present work, is obtained in Sect. 3, whereas for the other five clusters it is estimated from the radial density profiles given in the literature. The estimated values of $r_{\text {pre }}$ are given in the third column of Table 6 . The ratio $\left(r_{\text {lim }} / r_{\text {pre }}\right)$ is 
found to decrease with the age of the clusters and can be represented by an exponential law as

$r_{\lim } / r_{\text {pre }}=A \mathrm{e}^{B \log \tau}$

where $\tau$ is the age of the cluster.

The cluster will become gravitationally unbound when their radius reaches the limiting radius (i.e., ratio $=1$ ). An extrapolation of the present observational data indicate that these clusters need at least $\sim 18 \pm 5$ Gyr to attain the limiting radius. However this should be considered as the lower limit since $r_{\text {lim }}$ is calculated by taking into account the mass of presently observed stars only. The maximum time that a cluster can take to attain its limiting radius can be estimated if we take into account the unseen mass. Although it is difficult to estimate the total mass of the cluster, the contribution of unobserved faint cluster members in the estimation of the total mass of the cluster can be included if we assume that the mean slope of the MF is the same up to the lowest mass stars formed in the cluster and extrapolating the values to the lowest mass. We know that the slope of the MF at the fainter end is still an open question and in the present work we find evidence of turnover in the $\mathrm{MF}$ at $\sim 1 M_{\odot}$; however, for the sake of simplicity, we made this assumption. The ratio of $r_{\text {lim }}$ $\left(M_{\mathrm{L}}=0.1 M_{\odot}\right)$ and $r_{\text {pre }}$, shown in Fig. 6b, also decreases in the same manner as described earlier. The clusters having stars of $M_{\mathrm{L}}=0.1 M_{\odot}$ may take about $32 \pm 8 \mathrm{Gyr}$ to attain the limiting radius and this can be considered as an upper limit for the time to attain the limiting radius.

\section{Conclusions}

The radial structure of three clusters, namely King 5 , King 7 and Be 20 can be well explained by King's empirical model (1962), whereas the other two clusters (Be 64 and Be 69) show an irregular structure. A comparison of the radial density profile of these clusters indicates that evolution of the central region of the clusters is similar whereas the outer region of the clusters may be modified by dynamical evolutionary processes. CRDs of King 5 and King 7, where we could divide the sample into three mass ranges, indicate that the distribution of the low mass population of King 5 cannot be considered as different, which consequently suggests that a significant amount of energy equipartition has already taken place in the case of King 5 . The CRDs of the low mass population of King 7 can be considered as different at a confidence level $\sim 0.98$, indicating that equipartition of energy is still in progress.

Mass segregation, in the sense that massive stars tend to lie near the cluster center, is observed in the clusters King 5, King 7 and Be 20. The slopes of the MF of three clusters (Be 64, Be 69 and King 5) are in agreement with the Salpeter value. There is an indication of spatial variations of the MF in the two clusters King 5 and King 7. The cluster Be 20 shows a flat MF akin to MFs of older clusters. All the clusters are dynamically relaxed therefore the observed mass segregation is most likely due to the dynamical evolution process. It is interesting to note
Table 6. The cluster mass, present cluster radius $\left(r_{\text {pre }}\right)$, dynamical relaxation time $\left(T_{\mathrm{E}}\right)$ in years, cluster age $(\tau)$ in years, limiting radius and $\ln$ [ratio $\left.\left(r_{\mathrm{lim}} / r_{\mathrm{pre}}\right)\right]$ for the sample of five clusters used in the present study along with additional five clusters for which the data have been taken from the literature.

\begin{tabular}{|c|c|c|c|c|c|c|}
\hline Cluster & $\begin{array}{l}\text { Mass } \\
\left(M_{\odot}\right) \\
\end{array}$ & $\begin{array}{l}r_{\text {pre }} \\
(\mathrm{pc})\end{array}$ & $\begin{array}{c}\log \left(T_{\mathrm{E}}\right) \\
(\mathrm{yr})\end{array}$ & $\begin{array}{c}\log (\tau) \\
(\mathrm{yr})\end{array}$ & $\begin{array}{l}r_{\lim } \\
(\mathrm{pc})\end{array}$ & $\begin{array}{l}\ln \text { (ratio) } \\
\left(r_{\text {lim }} / r_{\text {pre }}\right)\end{array}$ \\
\hline Be $64^{1}$ & 78 & 1.41 & 6.4 & $8.9-9.0$ & 5.49 & $\overline{1.36 \pm 0.18}$ \\
\hline Be $69^{1}$ & 84 & 1.52 & 6.5 & $8.9-9.0$ & 5.59 & $1.30 \pm 0.20$ \\
\hline King $7^{2}$ & 351 & 1.87 & 6.7 & $8.8-8.9$ & 8.31 & $1.49 \pm 0.19$ \\
\hline King $5^{3}$ & 200 & 1.57 & 6.6 & 9.0 & 6.65 & $1.44 \pm 0.22$ \\
\hline Be $20^{3}$ & 198 & 6.57 & 7.6 & 9.7 & 11.24 & $0.54 \pm 0.15$ \\
\hline Be $81^{4}$ & 306 & 1.91 & - & 9.0 & 4.93 & $0.95 \pm 0.20$ \\
\hline Be $99^{4}$ & 177 & 3.84 & - & 9.5 & 7.46 & $0.66 \pm 0.18$ \\
\hline N $7044^{4}$ & 700 & 2.15 & - & 9.2 & 9.10 & $1.44 \pm 0.20$ \\
\hline $\operatorname{Tr} 5^{5}$ & 3000 & 10.01 & - & 9.6 & 18.39 & $0.61 \pm 0.18$ \\
\hline N $6791^{6}$ & 4070 & 10.82 & - & 9.95 & 14.47 & $0.29 \pm 0.18$ \\
\hline N $6791^{*}$ & & 9.70 & - & & & $0.40 \pm 0.20$ \\
\hline \multicolumn{7}{|c|}{$\begin{array}{ll}1 & \text { Pandey et al. (1997), } \\
2 & \text { Durgapal et al. (1997), } \\
3 & \text { Durgapal et al. }(2001), \\
4 & \text { Sagar \& Griffiths }(1998 \mathrm{a}), \\
5 & \text { Kaluzny (1998), } \\
6 & \text { Kaluzny \& Udalski }(1992) . \\
* & \text { In the literature } r_{\text {pre is given as } 10.82 \text { pc. We estimated }} \\
& r_{\text {pre }} \approx 9.70 \text { and used this value for the sake of uniformity. }\end{array}$} \\
\hline
\end{tabular}

that the ratio of the limiting radius to the present radius of the cluster decreases with the age of the cluster. The present study indicates that the clusters under discussion need a minimum time of about $18 \pm 5$ Gyr to attain their limiting radius.

Acknowledgements. The authors thank Prof. Ram Sagar for useful discussions. Thanks are also due to anonymous referee for useful comments.

\section{References}

Aparicio, A., Bertelli, G., Chiosi, C., \& Garcia-Pelayo, J. M. 1990, A\&A, 240, 262

Aparicio, A., Alfaro, E. J., Delgado, A. J., Rodriguez-Ulloa, J. A., \& Cabrera-Cano, J. 1993, AJ, 106, 1547

Bertelli, G., Bressan, A., Chiosi, C., Fagotto, F., \& Nasi, E. 1994, A\&AS, 106, 275

Carraro, G., \& Patat, F. 1995, A\&AS, 276, 563

Durgapal, A. K., Pandey, A. K., \& Mohan, V. 1997, BASI, 25, 489

Durgapal, A. K., Pandey, A. K., \& Mohan, V. 2001, A\&A, in press

Kaluzny, J. 1998, A\&AS, 133, 25

Kaluzny, J., \& Rucinski, S. M. 1995, A\&AS, 114, 1

Kaluzny, J., \& Udalski, A. 1992, AcA, 42, 29

Kholopov, P. N. 1969, Soviet Astr., 12, 625 
King, I. 1962, AJ, 67, 471

Landolt, A. U. 1983, AJ, 88, 439

Mateo, M. 1988, ApJ, 331, 261

Mathieu, R. D. 1985, Dynamics of star clusters, 113, 427, ed. J. Goodman, \& P. Hut

Mathieu, R. D., \& Latham, D. W. 1986, AJ, 92, 1364

McClure, R. D., VandenBerg, D. A., Smith, G. H., et al. 1986, ApJ, 307, L49

McNamara, B. J., \& Sekiguchi, K. 1986, ApJ, 310, 613

Meylan, G. 2000, to appear in proceedings of the Strasbourg Observatory workshop on Massive stellar clusters, ASP Conf. Ser. [Astro-ph/0003390]

Pandey, A. K., Bhatt, B. C., Mahra, H. S., \& Sagar, R. 1989, MNRAS, 236, 263

Pandey, A. K., Mahra, H. S., \& Sagar, R. 1992, BASI, 20, 295
Pandey, A. K., Durgapal, A. K., Bhatt, B. C., Mohan, V., \& Mahra, H. S. 1997, A\&AS, 122, 111

Pandey, A. K., Ogura, K., \& Sekiguchi, K. 2000, PASJ, 52, 847

Patat, F., \& Carraro, G. 1995, MNRAS, 272, 507

Sagar, R., Myakutin, V. I., Piskunov, A. E., \& Dluzhnevskaya, O. B. 1988, MNRAS, 234, 831

Sagar, R., \& Richtler, T. 1991, A\&A, 250, 324

Sagar, R., \& Griffiths, W. K. 1998a, MNRAS, 299, 1

Sagar, R., \& Griffiths, W. K. 1998b, MNRAS, 299, 777

Salpeter, E. E. 1955, ApJ, 121, 161

Scalo, J. 1986, Fund. Cosmic Phys., 11, 1

Scalo, J. 1998, The Stellar Initial Mass Function, ed. G. Gilmore, \& D. Howell, ASP Conf. Ser., 142, 201

Spitzer, L., \& Hart, M. H. 1971, ApJ, 164, 399

Stetson, P. B. 1987, PASP, 99, 191 